Wright State University

CORE Scholar

Special Session 5: Carbon and Oxide Based

Nanostructured Materials (2012)

Special Session 5

6-2012

\title{
AC Magnetic Heating of Superparamagnetic Fe and Co Nanoparticles
}

\author{
Anna Lukawska \\ Zafrullah Jagoo \\ Gregory Kozlowski \\ Wright State University - Main Campus, gregory.kozlowski@wright.edu \\ Zafer Turgut \\ Hiroyuki Kosai
}

See next page for additional authors

Follow this and additional works at: https://corescholar.libraries.wright.edu/ss5_2012

Part of the Physics Commons

\section{Repository Citation}

Lukawska, A., Jagoo, Z., Kozlowski, G., Turgut, Z., Kosai, H., Sheets, A., Bixel, T., Wheatley, A., Abdulkin, P., Knappett, B., Houlding, T., \& Degirmenci, V. (2012). AC Magnetic Heating of Superparamagnetic Fe and Co Nanoparticles. .

https://corescholar.libraries.wright.edu/ss5_2012/3

This Presentation is brought to you for free and open access by the Special Session 5 at CORE Scholar. It has been accepted for inclusion in Special Session 5: Carbon and Oxide Based Nanostructured Materials (2012) by an authorized administrator of CORE Scholar. For more information, please contact library-corescholar@wright.edu. 


\section{Authors}

Anna Lukawska, Zafrullah Jagoo, Gregory Kozlowski, Zafer Turgut, Hiroyuki Kosai, Alexander Sheets, Tyler Bixel, Andrew Wheatley, Pavel Abdulkin, Benjamin Knappett, Thomas Houlding, and Volkan Degirmenci 


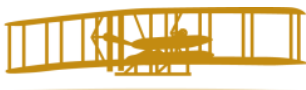

WRIGHT STATE UNIVERSITY
AC Magnetic Heating of

\section{Superparamagnetic $\mathrm{Fe}$ and $\mathrm{Co}$ Nanoparticles}

Anna Lukawska, Zafrullah Jagoo, and Gregory Kozlowski

Wright State University, Physics Department

3640 Col. Glenn Hwy., Dayton, OH 45435, USA

Zafer Turgut, Hiroyuki Kosai, Alexander Sheets, and Tyler Bixel Propulsion Directorate, AFRL, Wright-Patterson AFB, Ohio 45433, USA

Andrew Wheatley, Pavel Abdulkin, and Benjamin Knappett

Cambridge University, Chemistry Department, Lensfield Road, Cambridge CB2 1EW, UK

Thomas Houlding, and Volkan Degirmenci

Queen's University Belfast, School of Chemistry \& Chemical Engineering, Stranmillis

Road, Belfast BT9 5AG, Northern Ireland, UK

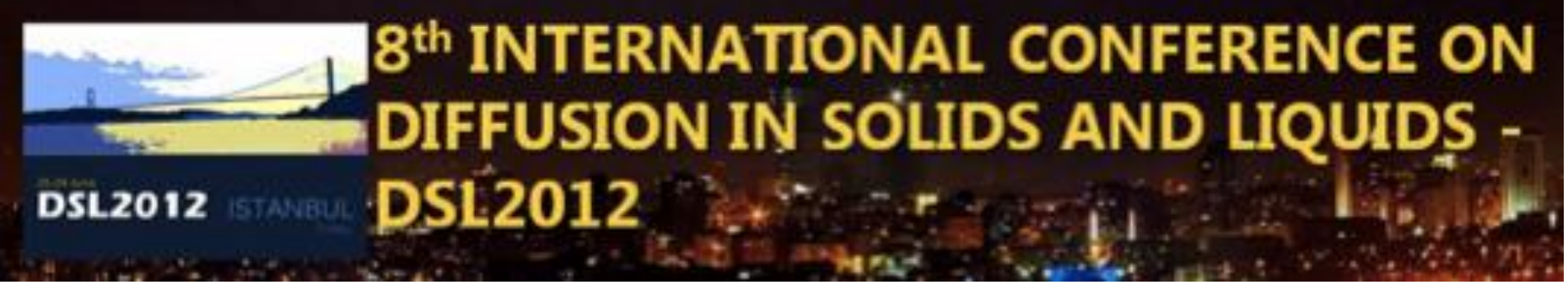




\section{Content}

- Introduction

- Magnetism

- Superparamagnetism

- Heating mechanisms

- System

- Analysis tools

- Fe

- Co

- Summary 


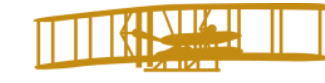 \\ WRIGHT STATE \\ UNIVERSITY}

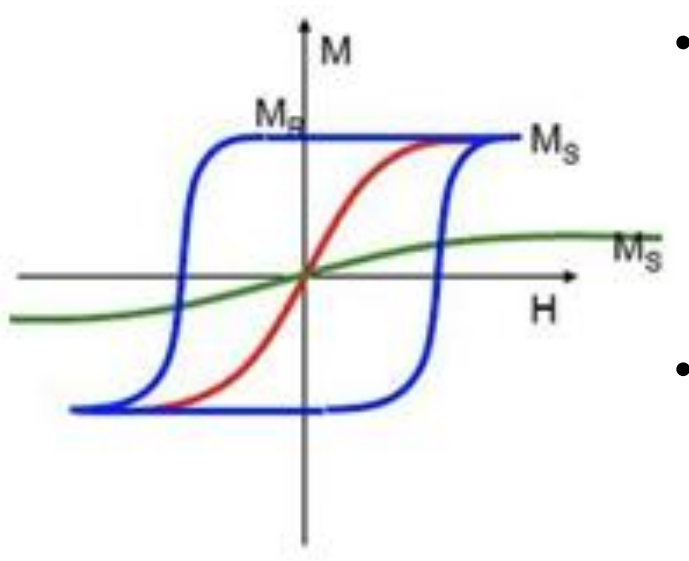

Ferromagnetism

Paramagnetism

Superparamagnetism

\section{Magnetism}

- Magnetic materials are classified by their susceptibility, $\boldsymbol{x}$ to magnetic fields:

- Diamagnetic - negative $X$,

- Paramagnetic - small and positive $X$,

- Ferromagnetic - large and positive $X$.

- In a large magnetic field the magnetic moments within the material align with the field, giving the saturation magnetization, $\mathbf{M}_{\mathbf{s}}$. As the magnitude of the field decreases the total magnetization decreases and reaches at zero field the remanent magnetization, $\mathbf{M}_{\mathbf{R}}$. The coercive field $\boldsymbol{H}_{c}$ must be applied to get back zero magnetization.

- Nanoparticles with diameter less than $100 \mathrm{~nm}$ have a single magnetic domain, a uniaxial anisotropy (the magnetic energy is the lowest when particles' magnetization has the same orientation as an anisotropy axis)

- Difference between maximum and minimum magnetic energies (magnetization pointing along the anisotropy axis and perpendicular to it) is:

$$
\Delta E=K V \sin ^{2} \theta
$$

where $\mathbf{K V}$ is an energy barrier, $\mathbf{K}$ is the anisotropy constant, and $\mathbf{V}$ is volume. 


\section{Superparamagnetism}

- KV value gets smaller as ferromagnetic nanoparticles $(<100 \mathrm{~nm})$ gets smaller, and at some point it is comparable with the thermal energy $\mathbf{k}_{\mathbf{B}} \mathbf{T}$.

- Assuming that particles are immobilized, this leads to a spontaneous random orientation of the magnetic moment inside the particles (zero remanence magnetization and coercivity, so no hysteresis).

- The magnetization will be zero until it will be biased in one direction by applied an external magnetic field. In this state the particle is superparamagnetic.

- The lifetime of particles magnetization (the time which the systems need to achieve zero magnetization after an external magnetic field is switched off), is given by Néel process

$$
\tau_{N}=\tau_{0} e^{\left(\frac{K V}{k_{B} T}\right)}
$$


- Disorder (high entropy) assembly of superparamagnetic nanoparticles (SN)

- Magnetized by magnetic field

- SN go to a highly ordered (low entropy) state

- Heat is added to the adiabatic system 


\section{Heating Mechanism (1)}

- The first thermodynamic law gives:

$$
d U=\delta W+\delta Q=\boldsymbol{H} d \boldsymbol{B}
$$

where, $U$ - the internal energy; $W$ - the magnetic work done on the system, $\boldsymbol{H}[A / m]$ - the magnetic field intensity, $\boldsymbol{B}[T]$ - the induction; $Q$ is the heat added to the system ( $\delta Q=0$, adiabatic process).

- Heating is a thermodynamic mechanism that relies on the fact that in a high-frequency magnetic field the nanoparticles magnetization is always trying to catch up with the applied filed.

- Since $\boldsymbol{H}$ and $\boldsymbol{B}$ are collinear, only magnitudes are required:

$B=\mu_{0} H+M$, where $\mu_{0}=4 \pi \cdot 10^{-7}[T m / A]$ is the permeability of free space. Thus, the change in the internal energy is:

$$
\Delta U=\mu_{0} \oint H d(H+M)=-\mu_{0} \oint M d H
$$

- When the magnetization lags the field, the integrand is negative, indicating that magnetic work is converted to internal energy. 


\section{Heating Mechanism (2)}

For ferrofluids the magnetization can be defined as a complex susceptibility: $\chi=\chi^{\prime}-i v^{\prime \prime}$ Since, $\chi=\frac{\chi_{0}}{1+i \omega \tau} \quad$ then $\chi^{\prime \prime}=\frac{\omega \tau \chi_{0}}{1+(\omega \tau)^{2}}$

The magnetic field intensity is: $H(t)=H_{0} \cos \omega t=\operatorname{Re}\left\{H_{0} e^{i \omega t}\right\}$ :

With $d H=-H_{0} \omega \sin \omega t$.

The magnetization is:

$M(t)=\chi H(t)=\operatorname{Re}\left\{\chi H_{0} e^{i \omega t}\right\}=\operatorname{Re}\left\{\left(\chi^{\prime}-i \chi^{\prime \prime}\right)(\cos \omega t+i \sin \omega t)\right\}=H_{0}\left(\chi^{\prime} \cos \omega t+\chi^{\prime \prime} \sin \omega t\right.$ Therefore, the change in internal energy is:

$$
\Delta U=\mu_{0} \omega H_{0}^{2} \chi^{\prime \prime} \int_{0}^{\frac{2 \pi}{\omega}} \sin ^{2} \omega t d t=\mu_{0} \omega H_{0}^{2} \chi^{\prime \prime} \frac{\pi}{\omega}=\mu_{0} H_{0}^{2} \chi^{\prime \prime} \pi
$$

The volumetric power $P$ generated by the assembly of nanoparticles and that heats surrounding environment is:

$P=f \pi \mu_{0} H_{0}^{2} \chi^{\prime \prime} \quad P=f \pi \mu_{0} H_{0}^{2} \frac{\omega \tau \chi_{0}}{1+(\omega \tau)^{2}}=f \pi \mu_{0} H_{0}^{2} \frac{2 \pi f \tau \chi_{0}}{1+(2 \pi f \tau)^{2}}$

where, $T$ is the effective relaxation time (due to Neel processes $\left.\boldsymbol{T}=\boldsymbol{T}_{N}\right)$. 


\section{System}

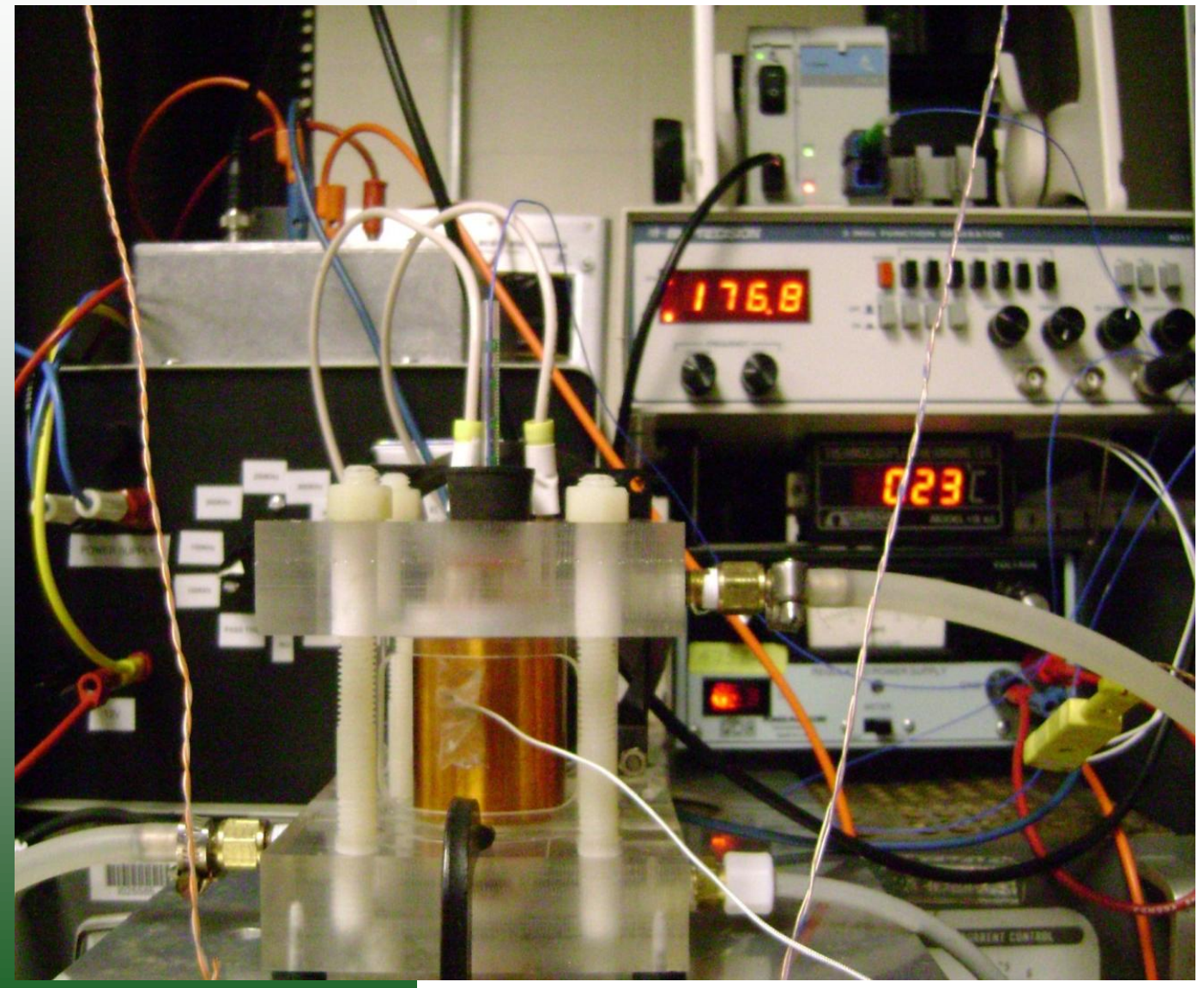

- A custom-made power supply

- A 20-turn insulated copper coil in the shape of a spiral solenoid

- Cooling is done with water

- A fiber-optic temperature sensor with an accuracy of $0.0001 \mathrm{~K}$

- At frequency $348 \mathrm{kHz}$ the magnetic field with magnitude of $20.6 \mu \mathrm{T}$ is generated inside the coil 


\section{Analysis tools}

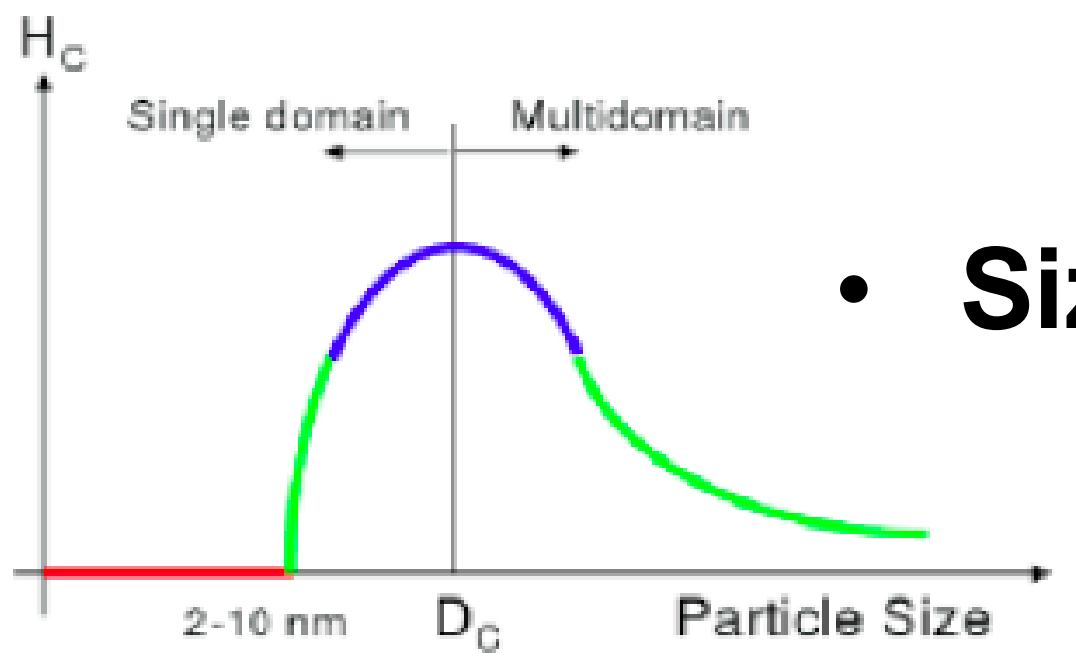

- SAR (Specific Absorption Rate) = SPL (Specific Power Loss) [W/g]

- Normalized SPL for different samples with different mass

$$
S P L^{\prime}=S P L / m\left[W / g^{\wedge} 2\right]
$$


- 5 different samples were measured

- Single-domain size limit: $15 \mathrm{~nm}$

- Superparamagnetic size limit: $6 \mathrm{~nm}$

- Heat capacity $\left[\mathrm{J} /{ }^{\circ} \mathrm{C}\right.$ g] $=0.4198$

\begin{tabular}{|c|c|c|c|c|c|c|}
\hline Nanoparticle & Diameter [nm] & Coating thickness [nm] & Coating & Coercivity [0e] & $S P L^{\prime}[W / g \wedge 2]$ & Type \\
\hline Co31 & 6.5 & $\sim 0.3-0.6$ & bis(2-ethylhexyl)sulfosuccinate & 0 & 0.351 & Superparamagnet \\
\hline Co51 & 7.3 & $\sim 1$ & Oleic acid+Dibutylamine & 0 & 0.378 & Superparamagnet \\
\hline Co41 & 8.2 & $<2$ & $\begin{array}{c}\text { Oleic acid + } \\
\text { Triphenylphosphine }\end{array}$ & 0 & 1.316 & Superparamagnet \\
\hline $\mathrm{Co1}$ & 8.7 & 1 & Oleic acid & $\geq 0$ & 0.176 & $\begin{array}{l}\text { Single-Domain } \\
\text { Ferromagnet }\end{array}$ \\
\hline Co21 & 20 & $\sim 1.6$ & PVP & 601 & 0.147 & $\begin{array}{l}\text { Multi-Domain } \\
\text { Ferromagnet }\end{array}$ \\
\hline
\end{tabular}


- The maximum specific power loss or the highest heating rate for Co magnetic nanoparticles was achieved for particles of $8.2 \mathrm{~nm}$ in diameter coated with oleic acid to avoid its oxidation.

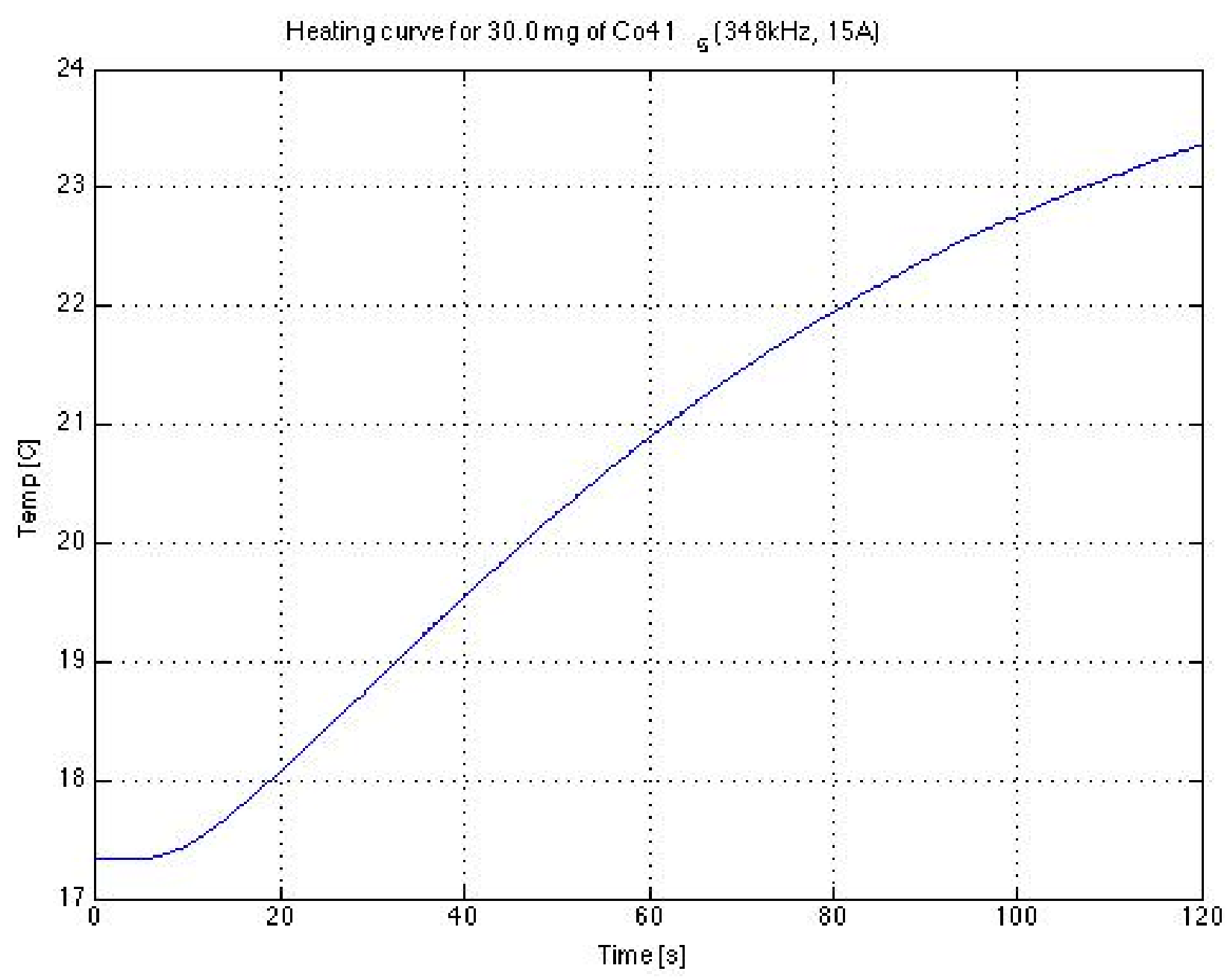


- 3 different samples were measured

- Single-domain size limit: 15nm

- Superparamagnetic size limit: 10nm

- Heat capacity $\left[\mathrm{J} /{ }^{\circ} \mathrm{C} \mathrm{g}\right]=0.4504$

\begin{tabular}{|c|c|c|c|c|c|c|}
\hline & Imeter [ & [Coating thickness [nm] & Coting 6 & chrity & $\mathrm{pl} / \mathrm{W}$ & Type \\
\hline Fet & 5.6 & ${ }^{N 1 \mathrm{nn}}$ & delecacad & 0 & 0.481 & Superparmagnet \\
\hline $\mathrm{Fet}$ & 11.3 & ${ }^{N 1 \mathrm{~mm}}$ & deicacid & 1 & 1.386 & Superparamagnet \\
\hline & 20 & N1nm & delecadod & $\geq 0$ & 0.344 & \\
\hline
\end{tabular}




\section{Summary}

- Those are preliminary results and they demand further investigation.

- Observed that Co with $8.2 \mathrm{~nm}$ diameter and Fe with $11.3 \mathrm{~nm}$ diameter in magnetic field of $20.6 \mu \mathrm{T}$, $(348 \mathrm{kHz}, 15 \mathrm{~A})$ gave the highest heating rates from investigated nanoparticles.

- One of next steps would be to immerse particles in fluid. 